\title{
Disseminated Nocardiosis in an Young Individual
}

\author{
Sarma $\mathbf{U}^{1 *}$, Dutta $\mathbf{R}^{2}$, Mishra $\mathbf{V}^{3}$, Pradhan $\mathrm{D}^{4}$, Singh $\mathrm{A}^{5}$ and Chakraborty $\mathrm{S}^{6}$ \\ ${ }^{1}$ Head of Critical Care Medicine, Nayati Medicity, India \\ ${ }^{2} \mathrm{Head}$ of Department of Thoracic Surgery, Nayati Medicity, India \\ ${ }^{3}$ Director of Critical care and Pulmonology, Nayati Medecity, India \\ ${ }^{4}$ Director of Lab Medicine, Nayati Medicity, India \\ ${ }^{5}$ Senior Consultant, Critical Care Medicine, Nayati Medecity, India \\ ${ }^{6}$ Senior Resident, Microbiology, Nayati Medecity, India
}

Case Report

Volume 5 Issue 1

Received Date: February 19, 2020

Published Date: March 13, 2020

DOI: $10.23880 /$ accmj-16000171

*Corresponding author: Utpal Sarma, Head of Critical Care Medicine, Nayati Medicity, NH 2, Mathura, UP, India, Tel: 8811079816; Email: drutpalrgci@gmail.com

\section{Abstract}

Disseminated nocardia infection is relatively uncommon and occurs in immune compromised Patient. The causative organism is an aerobic, gram-positive, branching, filamentous, weakly acid-fast bacteria. It can disseminate from pulmonary or cutaneous focus to virtually any organ. Herein, we are reporting a young individual with septic shock and multiple organ failure secondary to disseminated nocardiosis.

Keywords: Nocardia; Septic shock; Multiple organ failure

\section{Introduction}

Nocardia is an aerobic, gram-positive, branching, filamentous, weakly acid-fast bacterium. Nocardia can be found in soil, rotten plants, dust, or water and can infect humans via skin inoculation or respiratory inhalation [1]. Inhalation of the dust particles leads to pulmonary involvement commonly caused by $\mathrm{N}$. asteroids complex. Direct inoculation of the organism can lead to infections of the skin and subcutaneous tissue [2]. The reported mortality of disseminated nocardia infection is $24 \%$ [3].

\section{Case presentation}

A 19 years-old male admitted into the emergency department with fever, breathing difficulty, generalised body edema, and skin rashes for 10 days. On clinical evaluation, patient was having tachycardia (heart rate of $150 / \mathrm{min}$ ), tachypnoea (Breath rate 34/min), hypotension (BP 80/50 $\mathrm{mm} \mathrm{Hg}$ ) and fall in oxygen saturation $(96 \%$ on $6 \mathrm{~L} / \mathrm{min}$ oxygen through face mask). He was also having purulent skin discharge from skin lesions around the ear along with discrete purpuric spots all over the body. He has history of intramuscular injection of dexamethasone and pentazocin for 3 months.

He was resuscitated with intravenous fluid, oxygen and inotropic support. He required intubation and ventilatory support in view of unstable hemodynamics and breathing difficulty. Culture of tracheal aspirate, paired blood culture and discharge from skin lesion done to identify the causative organism. His initial chestX-ray shows bilateral lunginfiltrates (Figure 1). Ultrasonography of abomen shows grade 1 fatty liver and mild pleural effusion. Blood biochemistry showed $\mathrm{Hb} 12.9 \mathrm{gm} / \mathrm{dl}$, Total leucocyte count 12.1 thousands/ dl , platelets 10 thousands/cu mm of blood, INR 1.59, serum albumin $2.1 \mathrm{mg} / \mathrm{dl}$. Arterial blood gas analysis at emergency departement was pH 7.49, pO2 74, pCO2 24.1, HCO3 18.1 $\mathrm{mmol} / \mathrm{L}$, lactate value of $3 \mathrm{mmol} / \mathrm{L}$. Hemoglobin A1c $8.9 \%$ (normal range < 5.7), urine ketone positive, random blood sugar $88 \mathrm{mg} / \mathrm{dl}$. Provisional clinical diagnosis of community acquired pneumonia with septic shock was made. He was also worked up for tropical fever syndrome. Dengue serology, peripheral blood smear for malaria parasite was negative. 


\section{Anaesthesia \& Critical Care Medicine Journal}

He was treated with broad spectrum antibiotics, inotropic support (Nor adrenaline @ $0.4 \mathrm{mcg} / \mathrm{kg} / \mathrm{min}$ ) and ventilatory support at medical intensive care unit. One unit of Single donor pool platelet was transfused due to low platelate level. ET secretion was also sent for H1N1 PCR (Pollymerase chain reaction) which came as negative. Gram stain from tracheal aspirates showed plenty of thin, branching, filaments, weakly acid fast bacteria resembling nocardia species (Figure 2a). Blood culture was positive for nocardia species (Figure 2b). MRSA grew from the the discharges near left ear. Inj Ceftriaxone 1 gm @ 8th hourly \& Inj imepenum 500 @ 6 hourly was started for nocardia infection on day 4 of icu admission(once nocardia was isolated). Inj Clindamycin 600 mg @ 8th hourly was continued for MRSA grown in culture of discharges from the skin according to sensitivity result. Patient continued a downhill clinical course inspite of appropriate antibiotic coverage, respiratory and hemodynamic support. On day4 of hospital stay, blood investigation shows $\mathrm{Hb} 9.4 \mathrm{gm} / \mathrm{dl}$, TLC 2.3 thousands/cumm, Platelet 16 thousands. Day-5, patient developed alveolar haemorrhage leading to difficult ventilation succumbed to death.
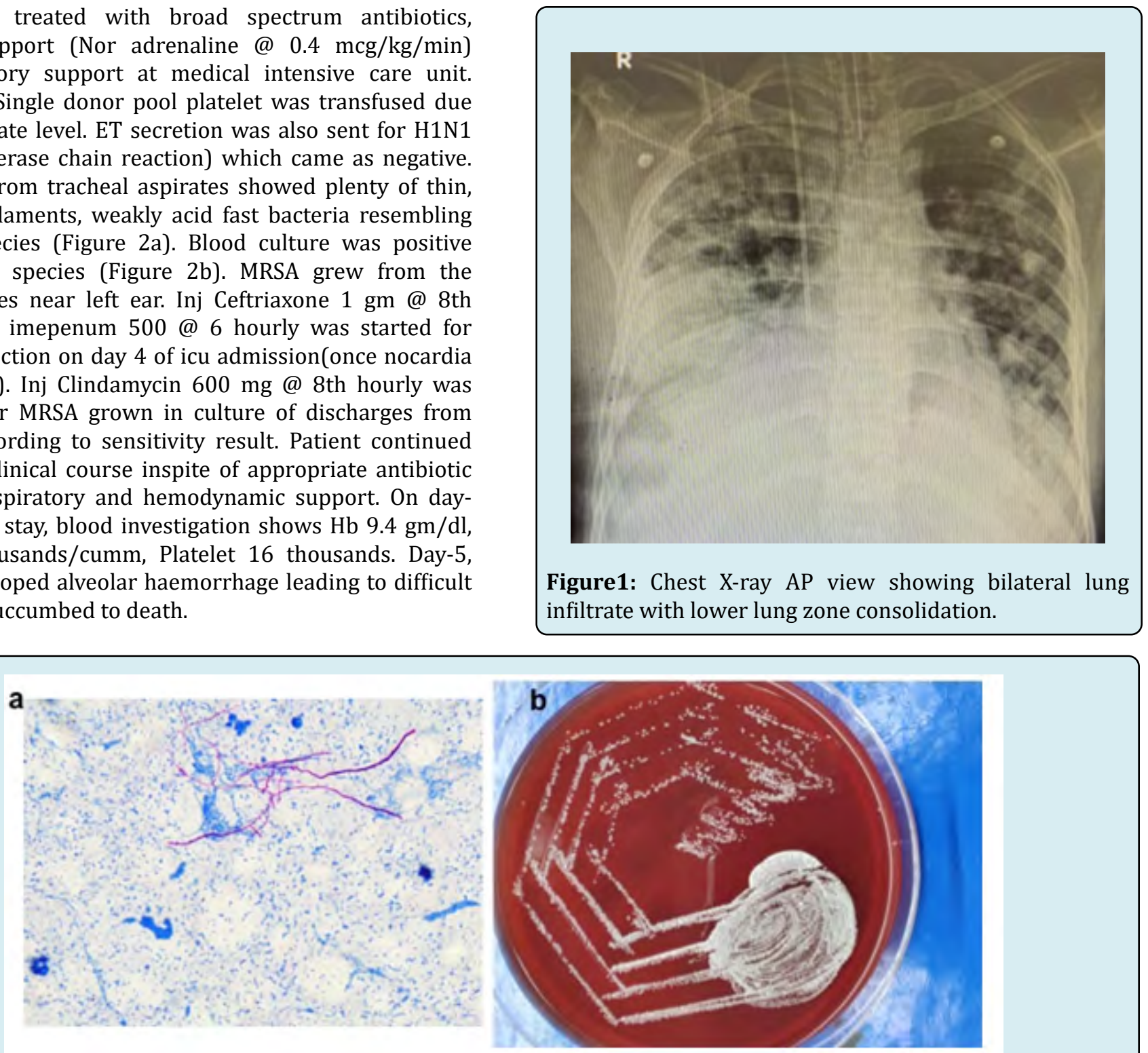

Figure 2a: Photomicrograph of Gram stain slide of tracheal aspirates shows plenty of thin, branching, filaments, weakly acid fast bacteria resembling nocardia species and Culture in blood agar.

Figure 2b: shows creamy white pitting colony of nocardia.

\section{Discussion}

Nocardiosis can present as an opportunistic infection in immune compromised patients specifically organ transplants patients on immunosuppressive medicine, patient on long standing steroid therapy. It can present as cutaneous infection, pulmonary infection and rarely disseminated infection with multiple organ involvement. Tan, et al. [4] in a retrospective analysis reported that cutaneous infection (56.6\%), pulmonary infection $(33.6 \%)$ and disseminated infection (7.1\%). The incidence rate of nocardiosis is considered to be increasing, due to immunosuppressing agents prescribed to patients with chronic lung disease, malignancy, or autoimmune disease as well as transplantation recipients [5].

Nocardia spp. are capable of causing disseminated disease that can readily enter the blood stream and spread throughout the body, including the skin, lung, central nervous system and abdominal organs [6]. Disseminated nocardiosis is a rare disease. It is advised to get contrast-enhanced CT abdomen to detect a liver abscess, and cranial MRI to find 


\section{Anaesthesia \& Critical Care Medicine Journal}

out structural disease. These tests are done to confirm the diagnosis of disseminated nocardiosis of the subcutaneous soft tissue, lungs and liver. Presence of nocardia infection in the brain, two or more non-contiguous organs, or in the blood was required for diagnosis of disseminated nocardiosis [7]. In our case both blood and tracheal aspirate showed the growth of Nocardia species. In our case, we initially made the differential diagnosis of tropical fever syndrome, as there were rashes, thrombocytopenia, and mild transaminase elevation. Injection doxycycline was started empirically to cover scrub typhus.

The primary regimens for nocardia pneumonia is the dual therapy trimethoprim plus sulpfmethoxazole (TMP-SMX) 15 $\mathrm{mg} / \mathrm{kg}$ body and imepenum-cillastin $500 \mathrm{mg}$ iv 6 th hourly. Inj Amikacin can be used as alternative therapy along with imepenum-cillastine. For skin abscess and lymphadenitis, the requirement of TMP-SMX is $5-10 \mathrm{mg} / \mathrm{kg} /$ day. In our case as there was thrombocytopenia we did not start TMP-SMX. Hence, the patient was treated with inj Ceftriaxone along with imepenum-cillastine. Tab Minocycline is an alternative to TMP-SMX at a dose of 100 to $200 \mathrm{mg}$ twice daily in case of skin abscess [8]. Linezolid, an oxazolidinone, is quite active against virtually all known pathogenic Nocardia species and has successfully been used in treatment of patients with disseminated and CNS nocardiosis [9].

Zia, et al. [10] in a series of 55 patients in a single institution found that the most common risk factor is chronic steroid therapy $(\mathrm{n}=38 ; 69.1 \%)$. Complications were observed in $32(58.2 \%)$ patients with septicaemia and respiratory failure being the most common $(n=15 ; 46.8 \%$ in each). Disseminated Nocardia infection occurred in 10 (31.2\%) patients. Majority of the patients required critical and lifesaving interventions. The most frequent of these was endotracheal tube placement for mechanical ventilation secondary to respiratory failure in $15(42.8 \%)$. The mortality rate of Nocardia is $34.5 \%(n=19)$. Our patient came in a critically ill state that required mechanical ventilation and inotropic support in intensive care unit.

\section{Conclusion}

Because of low incidence often it takes time to diagnose disseminated Nocardia infection. It is important that
Nocardiosis is ruled out in an immune compromised patient with pulmonary infiltrate with an indolent course.

\section{References}

1. Beaman BL, Beaman L (1994) Nocardia species: hostparasite relationships. Clin Microbiol Rev 7(2): 213-264.

2. Wilson JW (2012) Nocardiosis: Updates and clinical overview. Mayo Clin Proc 87(4): 403-407.

3. Wang HK, Sheng WH, Hung CC, Chen YC, Lee MH, et al. (2015) Clinical characteristic, microbiology, and outcomes for patients with lung and disseminated nocardiosis in a tertiary hospital. J Formos Med Assoc 114(8): 742-749.

4. Tan CK, Lai CC, Lin $\mathrm{SH}$, Liao $\mathrm{CH}$, Chou $\mathrm{CH}$, et al. (2010) Clinical and microbiological characteristics of Nocardiosis including those caused by emerging Nocardia species in Taiwan, 1998-2008. Clin Microbiol Infect 16(7): 966-972.

5. Liu WL, Lai CC, Ko WC, Chen YH, Tang HJ, et al. (2011) Clinical and microbiological characteristics of infections caused by various Nocardia species in Taiwan: a multicenter study from 1998 to 2010. Eur J Clin Microbiol Infect Dis 30(11): 1341-1347.

6. Shahapur PR, Peerapur BV, Shahapur RP, Honnutagi RM, Biradar MS (2014) Lymphocutaneous nocardiosis caused by Nocardia otitidiscaviarum: A case report and review of literature. J Nat Sci Biol Med 5(1): 197-201.

7. Lerner PI (1996) Nocardiosis. Clin Infect Dis 22 (6): 891903.

8. Sanford guide to Anti-microbial therapy, 2018.

9. Moylett EH, Pacheco SE, Brown-Elliott BA, Perry TR, Buescher ES, et al. (2003) Clinical experience with linezolid for the treatment of Nocardia infection. Clin Infect Dis 36(3): 313-318.

10. Zia K, Nafees T, Faizan M, Salam O, Asad SI, et al. (2019) Ten Year Review of Pulmonary Nocardiosis: A Series of 55 Cases. Cureus 11(5): e4759. 